\title{
Control and Application of Carbon Quantum Dots
}

\author{
Rohini V S \\ Department of Physics, Nrupatunga University, Bangalore, Karnataka, India \\ neelarohini@gmail.com
}

\begin{abstract}
Carbon quantum dots (CQDs) are a new class of small carbon nanoparticles with a particle size of less than 10 nm and have vast applications in the field of bioimaging, biosensing and disease- detection. These are promising materials for nano-biotechnology since it has smaller particle size, excellent biocompatibility and excitation wavelength dependent photoluminescence (PL) behavior, photo induced electron transfer, chemical inertness and low toxicity. These materials have excellent fluorescent properties such as broad excitation spectra, narrow and tunable emission spectra, and high photostability against photo bleaching and blinking than other fluorescent semiconductor quantumdots.
\end{abstract}

Keywords: quantum dots, photoluminescence (PL), emission spectra, CQDs

\section{Introduction}

\subsection{Theory of carbon dots}

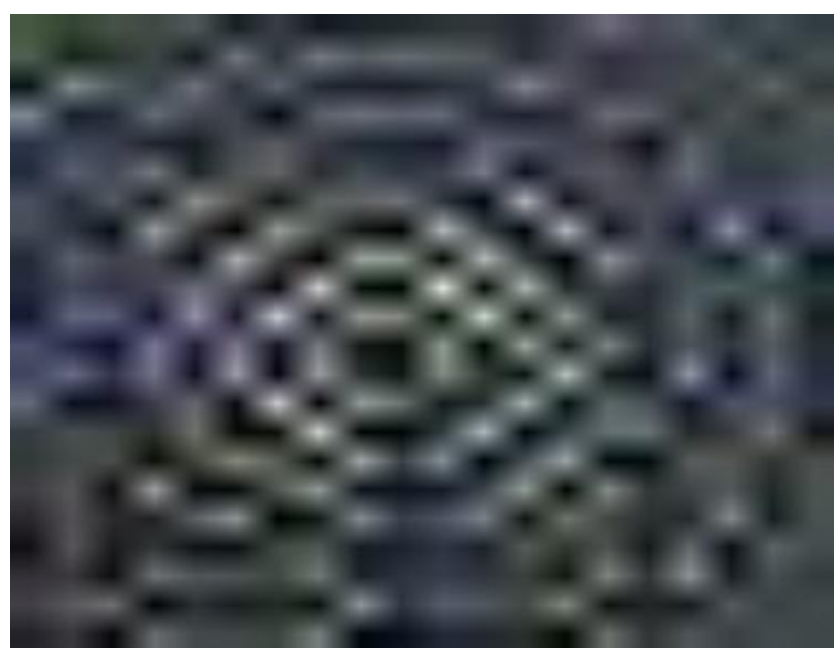

(Structure of Hydrolic CQD)

Carbon quantum dots (CQDs) are first discovered in 2004 during the purification of single walled carbon nanotubes. Generally Carbon Quantum dots (CQDs, C-dots or CDs) are small carbon nanoparticles (less than $10 \mathrm{~nm}$ in size) with some form of surface passivation.

Quantum dots are semiconductor particles with sizes of a few nm. QD emit light of a specific wavelength when a current is applied or exposed to light. Nanoparticles are also small structures but larger than QDs, usually ranging from 8-100nm. CQDs possess very strong and tunable fluorescence properties which enable their applications in biomedicines, optronics, sensors and catalytic applications. It has excellent photostability, small size, highly tunable photoluminescence (PL) property, biocompatibility, electrochemiluminescence, exceptional multi-photon excitation property. These materials can be functionalized with biomolecules and are less toxic and chemically inert for which these are used as effective carriers for drug delivery, biological imaging. CQDs also have promising applications in sensors, optronics and electrochemical luminescence.

Conventionally, CQDs are prepared by the surface functionalization of carbon nanoparticle with organic and polymeric molecules. Most of the preparation methods have followed the carbonization of carbon containing precursors. CQD synthesized by this method has widely varying optical properties but less controllable. The carbonization of various fruit juices, watermelon or pomelo peels, many food items, grass and plant leaves have produced CQDs. Chitosan is also used as a precursor for synthesis of CQD by hydrothermal carbonization. Various techniques of synthesis of carbon quantum dots, such as ultrasonic methods, hydrothermal treatment, laser ablation of graphite and microwave-assisted synthesis, strong acidic and electrochemical oxidation, pyrolysis of glycerol, exfoliation of graphite in organic solvent by modified Hummer's method, thermal carbonization of molecules as well as atmospheric plasma- based synthesis have been reported. The precursors are widely varied as sweet pepper, capsicum, watermelon peel, carbon sooth and carrot and synthetic polymer have been reported. The advantages of using natural resources for the synthesis of CQDs are cost-effective and eco-friendly. The green synthesis methods are highly acceptable as compared to physical and chemical processes. The numerous waste organic products are also used for the preparation of CQDs.

CQDs have vast biomedical and biotechnological applications like;

1) Drug delivery

2) Biosensors-Biosensors

3) Cell imaging-Live cell imaging

4) Biomedical imaging-Biomedical image processing

5) Targeted therapy

6) Bioanalytical assay

7) vivo animal targeting

8) Cancer diagnosis/therapy-

9) Ex-vivo live cell imaging 


\section{Green Synthesis (using natural resources)}

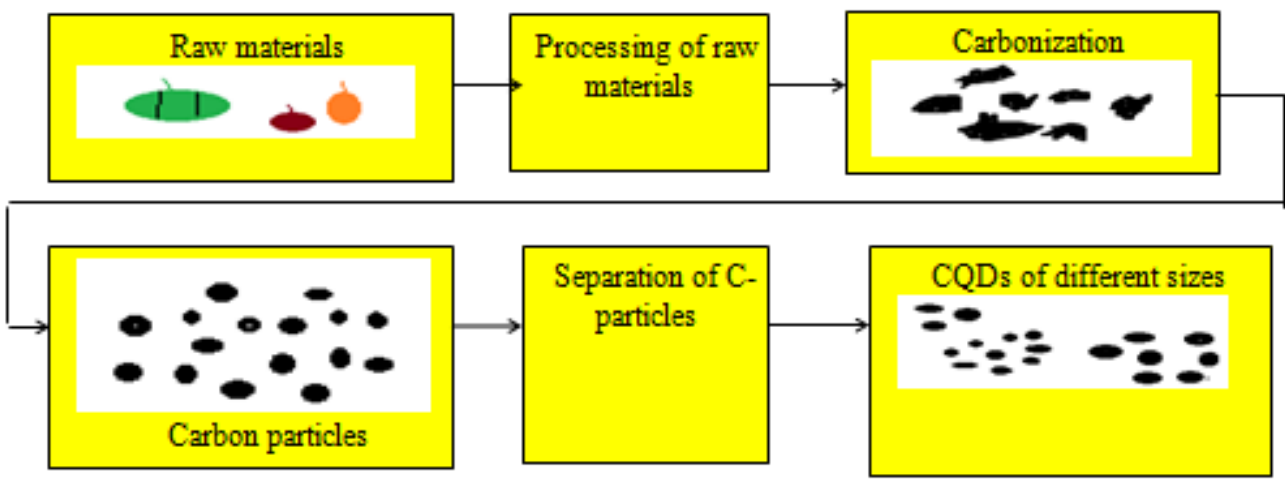

\subsection{Preparation of CQDs}

The natural resource is subjected to cutting, grinding, low flame hydrothermal heating, micro oven processing and carbonization on a high flme till it gets converted into fine carbon powder. This powder is filtered and carbon powder of different sized carbon grains are separated and labelled as size1, 2, 3 as shown below. Natural resource used is capsicum, carrot, palmoil, water melon
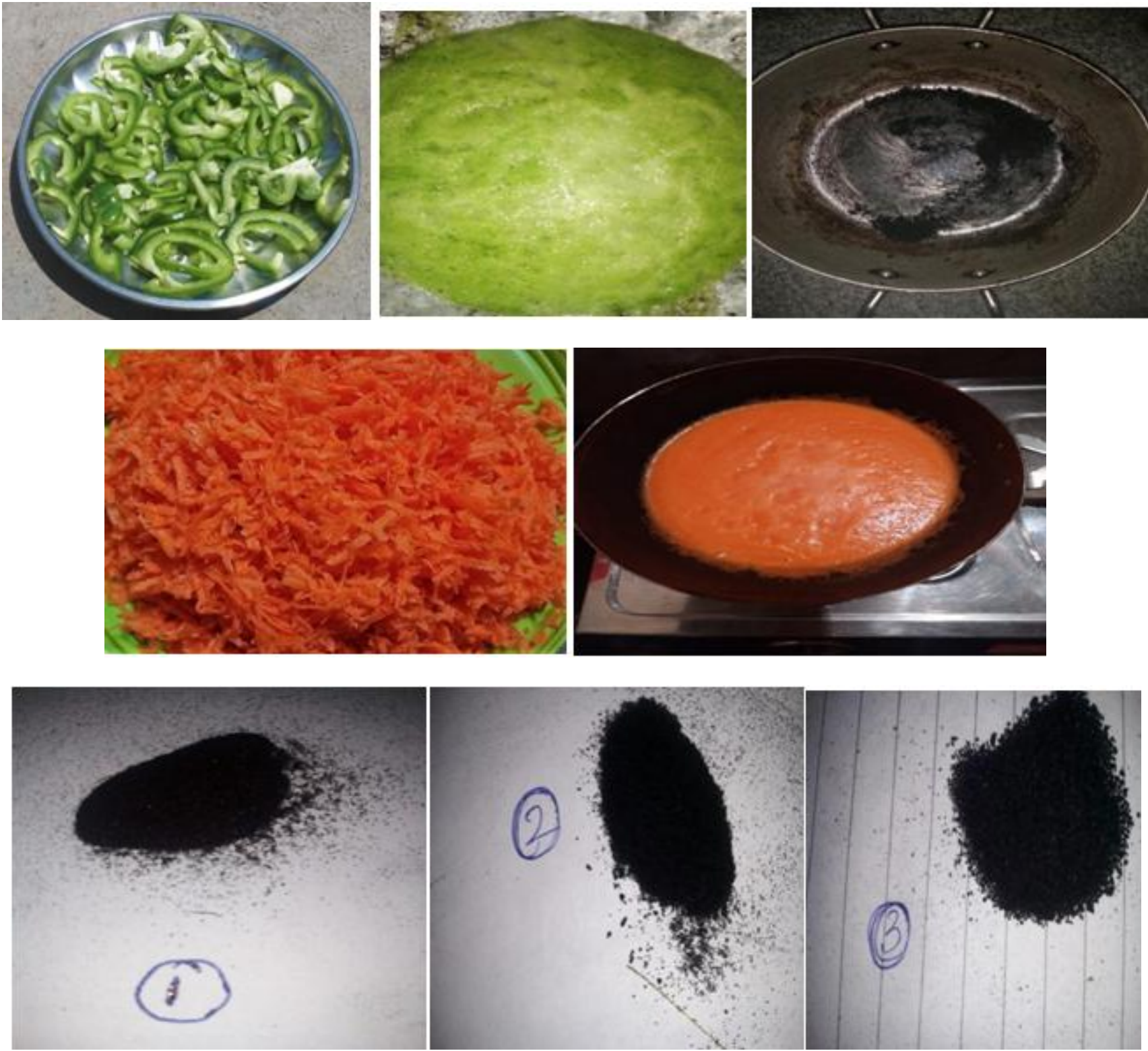


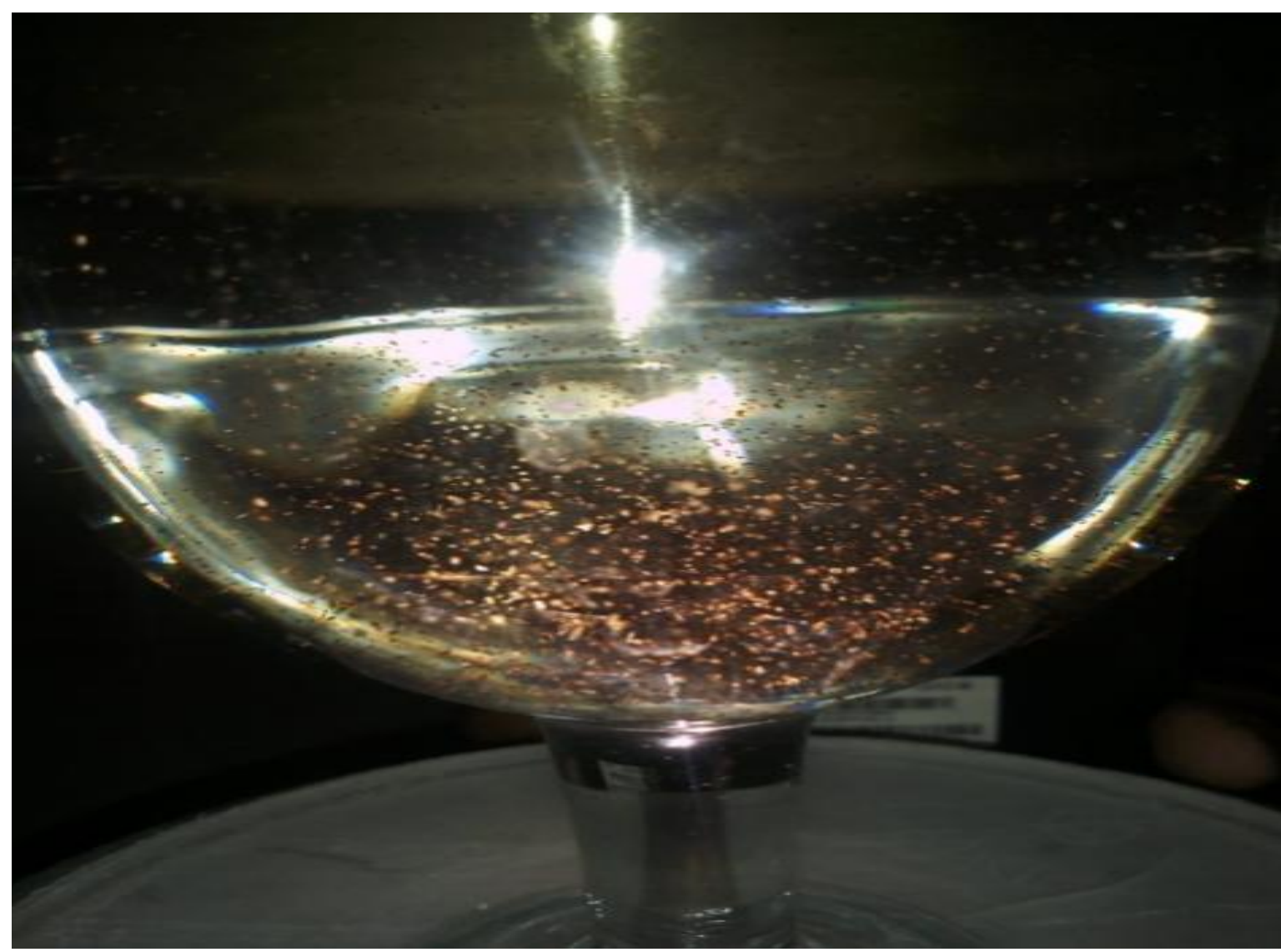

Hydrated carbon sample

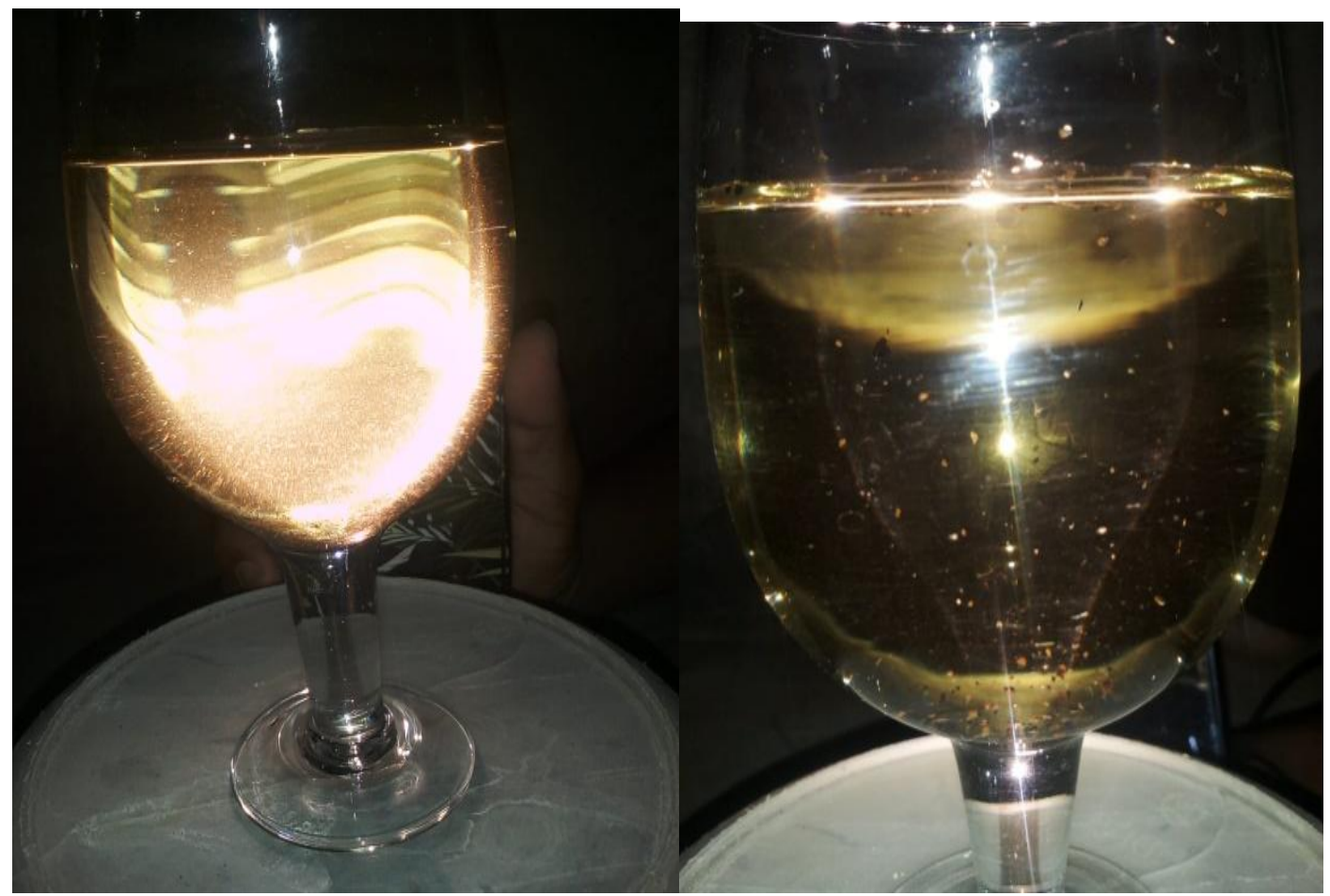

Hydrated carbon sample showing photoluminence with white light. Here onwards these hydrated carbonparticles are referred as hydrated hydraulic carbon quantum dots (HCQDs) and their special features are studied 


\subsection{Charecterization of CQDs}
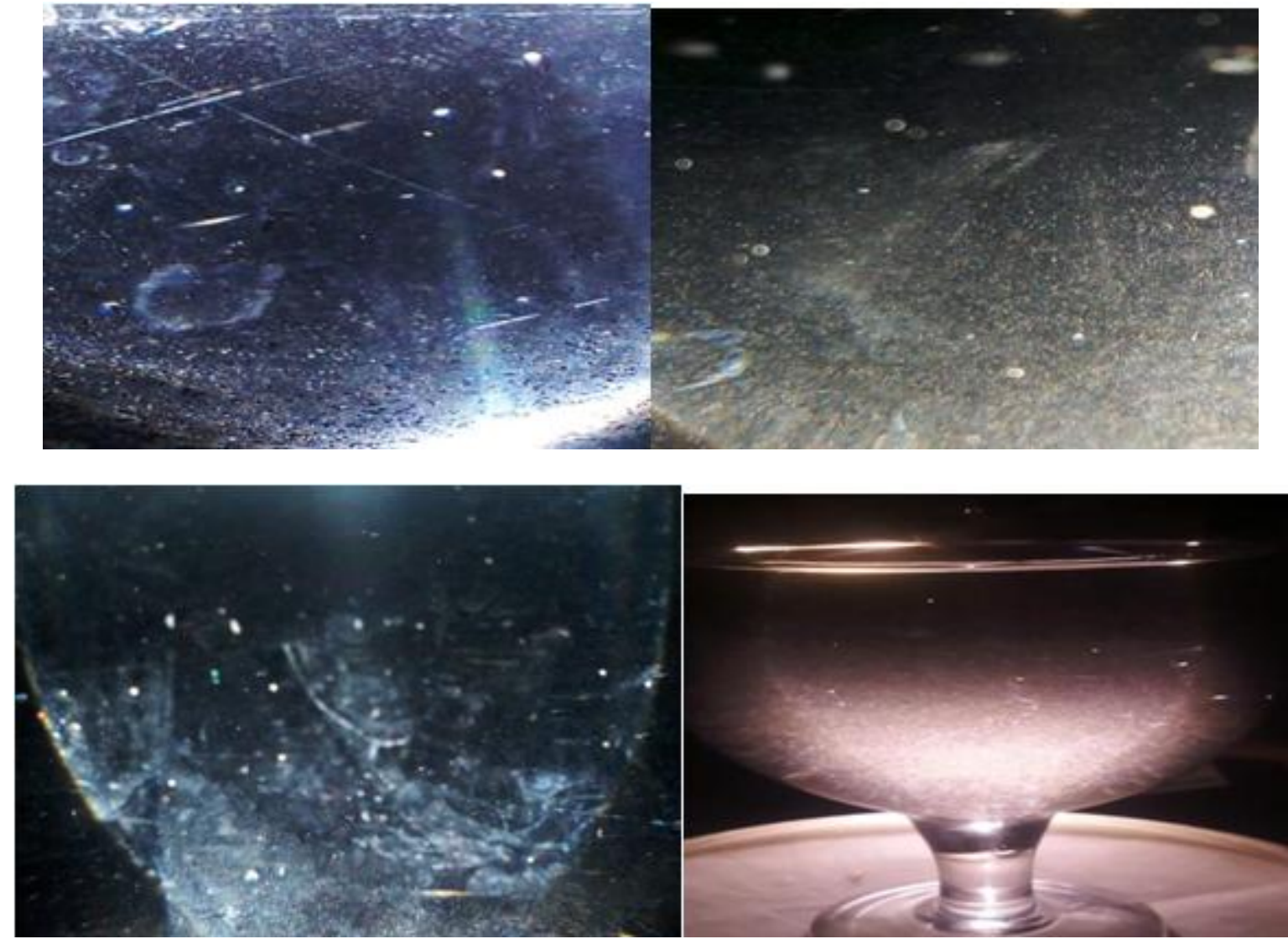

Enlarged view of hydrated CQDs (HCQDs)

When illuminated with white light, very small minute balls are seen floating/circulating in water which are HCQDs. An enlarged view of a single ball is shown below

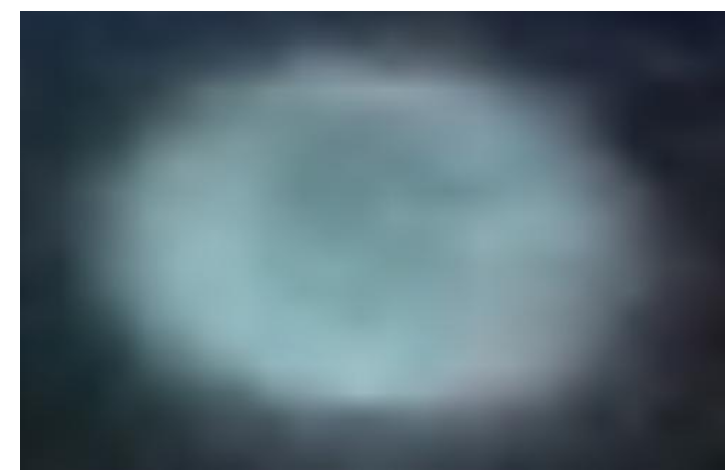

Single hydrulic CQD

When surface of this HCQD is enlarged and seen it looks like this. Enlarged HCQD shows ring like structures

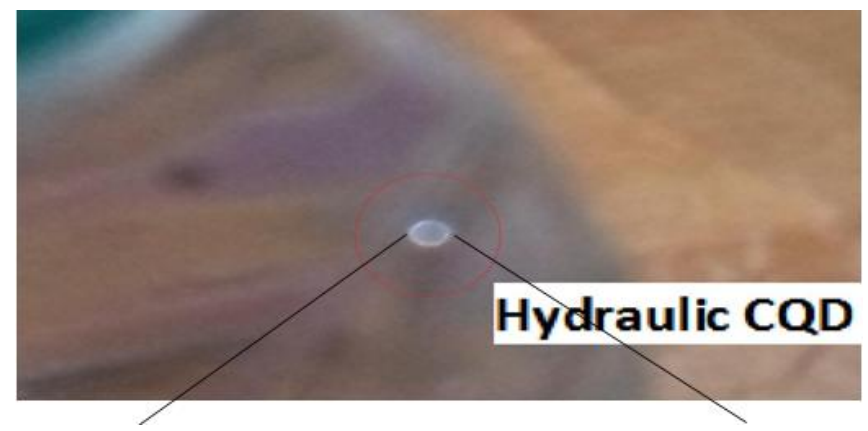

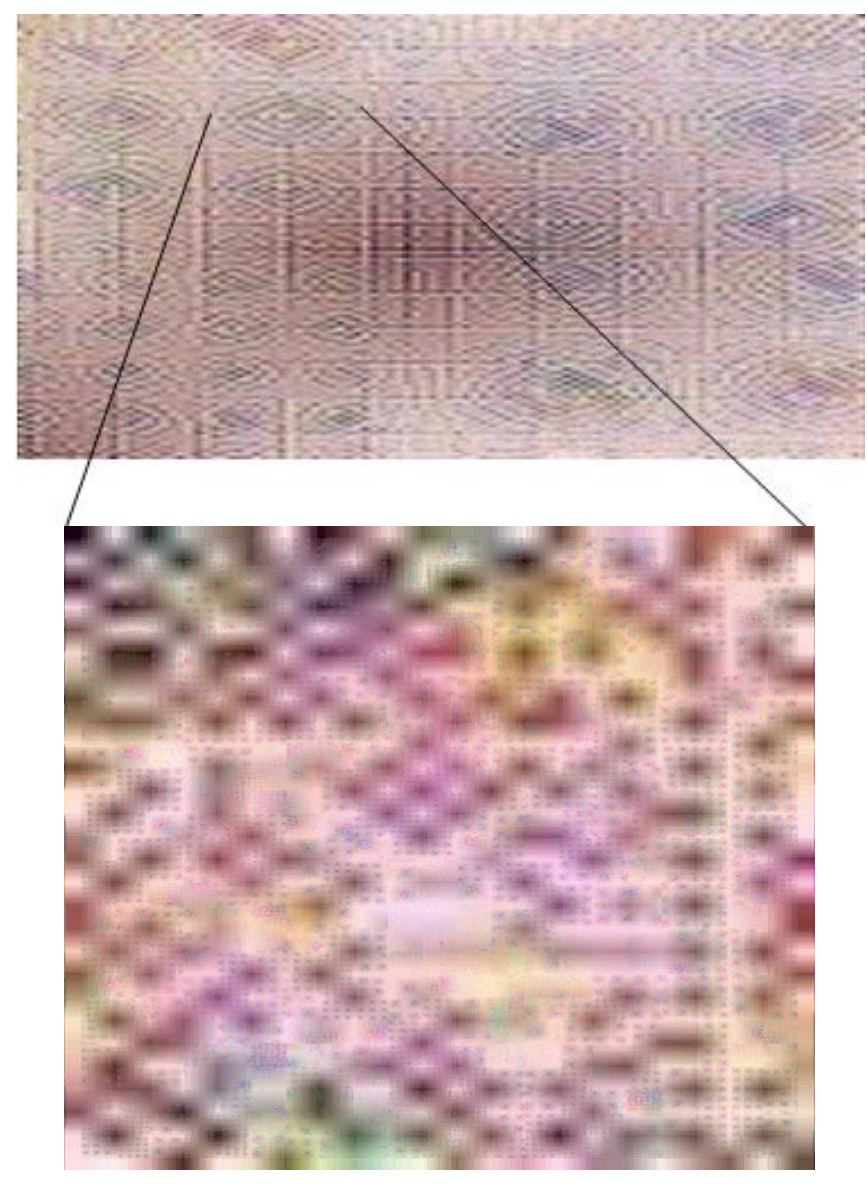




\section{Magnified image of surface of a HCQD}
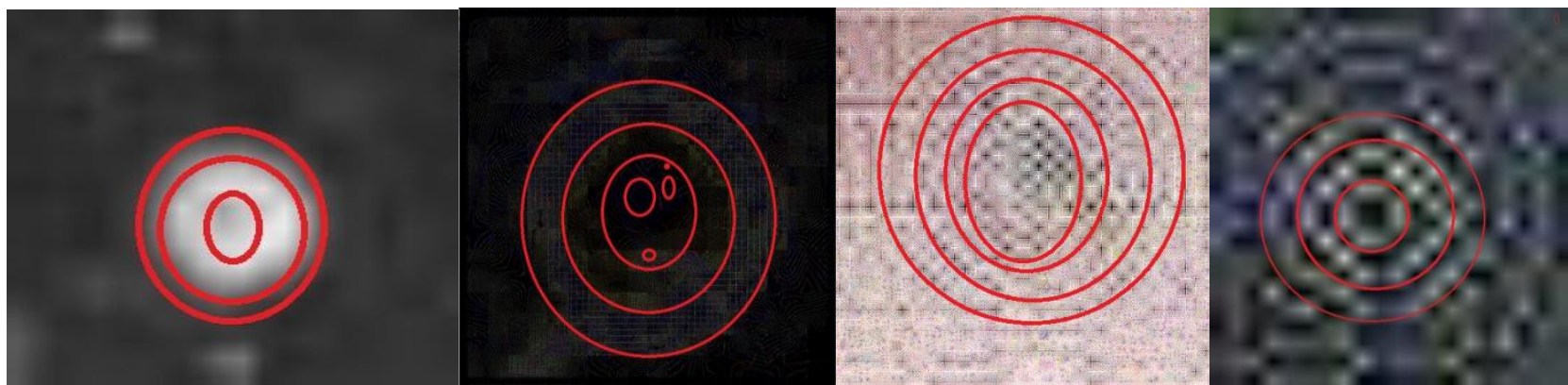

\subsection{Determination of size of HCQD}

\section{Part 1: Measurement of diameter of Hydraulic CQDs}
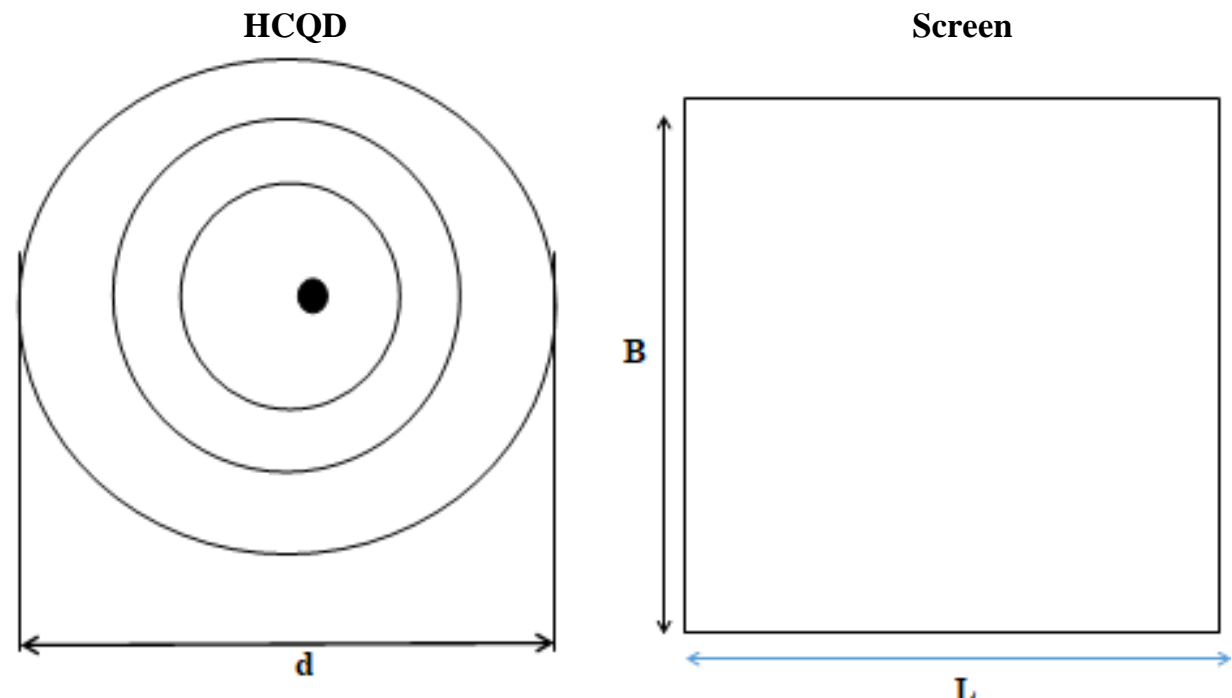

$\mathrm{L}=6.9 \times 10^{-2} \mathrm{~m}$; length $=3264 \mathrm{pxs} ; \mathbf{C}_{\mathbf{L}}=3264 / 6.9 \times 10^{-2}=$ $473.0435 \times 10^{2}=47304 \mathrm{pxs} / \mathrm{m}$

$\mathrm{B}=6.3 \times 10^{-2} \mathrm{~m}$; breadth $=2448 \mathrm{pxs}$

Determination of diameter of the circular rings (first magnification method)

$\mathrm{A}=3264 \times 2448 \mathrm{pxs}=7.990272 \times 10^{6} \mathrm{pxs}$

$\mathrm{A}\left(\mathrm{m}^{2}\right)=\left(6.9 \times 10^{-2} \mathrm{~m}\right) \times\left(6.3 \times 10^{-2} \mathrm{~m}\right)$

$\mathrm{A}\left(\mathrm{m}^{2}\right)=43.47 \times 10^{-4} \mathrm{~m}^{2}$

$\mathbf{C}_{\mathrm{A}}=\underline{\mathrm{A}(\mathrm{pxs})}=\underline{(3.264 \times 2.448) \times 10^{6} \mathrm{~m}}=0.1838 \times 10^{10} \mathrm{pxs} / \mathrm{m}^{2}$. $\mathrm{A}\left(\mathrm{m}^{2}\right) \quad 43.47 \times 10^{-4} \mathrm{~m}^{2}$

$$
\begin{gathered}
C_{A}=0.1838 \times 10^{10} \mathrm{pxs} / \mathrm{m}^{2} \\
C_{L}=4.7304 \times 10^{4} \mathrm{pxs} / \mathrm{m}
\end{gathered}
$$

\section{Calibration}

$\mathrm{D}($ diameter $)=$ (m) $\times \mathrm{C}_{\mathrm{L}}=$ ..pxs

\begin{tabular}{|c|c|c|c|c|c|c|}
\hline Ring no & $\begin{array}{c}\mathrm{D}_{1} \\
(\mathrm{~cm})\end{array}$ & $\begin{array}{c}\mathrm{D}_{1} \mathrm{px} \\
\left(\mathrm{x} 10^{3}\right)\end{array}$ & $\begin{array}{c}\mathrm{D}_{2} \\
(\mathrm{~cm})\end{array}$ & $\begin{array}{c}\mathrm{D}_{2} \mathrm{px} \\
\left(\mathrm{x} 10^{3}\right)\end{array}$ & $\begin{array}{c}\mathrm{D} 3 \\
(\mathrm{~cm})\end{array}$ & $\begin{array}{c}\mathrm{D}_{3} \mathrm{px} \\
\left(\mathrm{x} 10^{3}\right)\end{array}$ \\
\hline $\mathrm{I}$ & 0.5 & 3.1 & 0.7 & 4.34 & 1.1 & 6.82 \\
\hline II & 0.4 & 2.48 & 0.5 & 3.1 & 0.8 & 4.96 \\
\hline III & 0.4 & 2.48 & 0.7 & 4.34 & 1.2 & 7.44 \\
\hline MEAN & D1=0.0045m & $0.0006 \mathrm{~m}$ & & $0.00103 \mathrm{~m}$ & \\
Size of CQD (1 $1^{\text {st }}$ magnification $)=\mathrm{X}_{1}=\mathrm{t}(\mathrm{m}) / \mathrm{M}_{1}=1400 \mu \mathrm{m}$ \\
Size of CQD (2 ${ }^{\text {nd }}$ magnification $)=\mathrm{X} 2=\mathrm{t}(\mathrm{m}) /$ \\
1440000 $=0.9722 \mathrm{~nm}$
\end{tabular}

Where $\mathrm{M}_{1}=1, \mathrm{M}_{2}=144000$ 


\section{Graph 1}
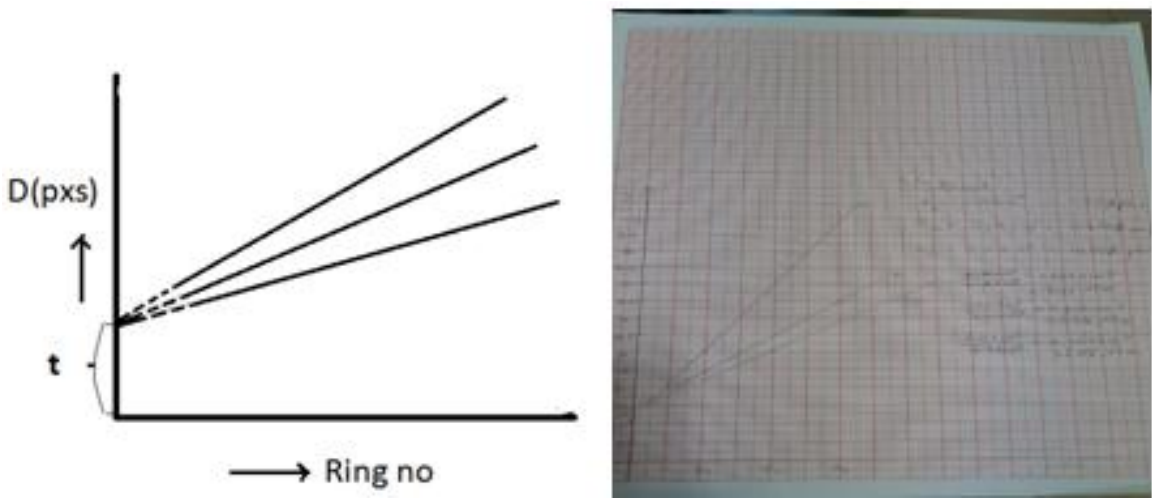

Size of $\mathrm{CQD}=\mathrm{x}_{\mathrm{i}}=\mathrm{t} / \mathrm{M}_{\mathrm{i}}$;

where $\mathrm{M}_{\mathrm{i}}$ is magnification factor for the ith magnification

\section{Part 2:- Optical characterization}

\section{A. Using Beer-Lambert Law.}

Required Apparatus:- Carbon quantum dots(particles of size1), distilled water, Beer Lamberts' instrument, test tube.
Principle: The Beer-Lambert law states that there is a linear relationship between the concentration and the absorbance of the solution, which enables the concentration of a solution to be calculated by measuring its absorbance.

\section{Diagram}

(water+CQDs)

filter

Incident light $\left(\mathrm{I}_{0)}\right.$ transmitted light $(\mathrm{I})$

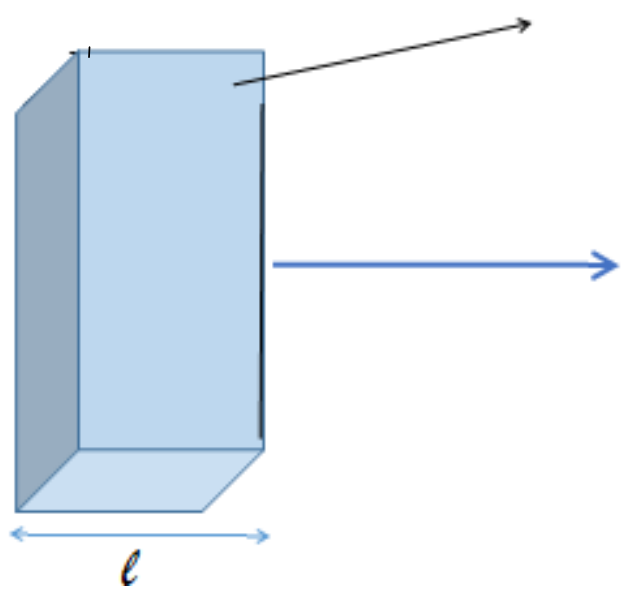

Table 1: Callibration graph for distilled water

\begin{tabular}{|c|c|c|}
\hline S. No & Intensity $(\mu \mathrm{A})$ & Wavelength $\lambda\left(\mathrm{A}^{0}\right)$ \\
\hline 1 & 2.7 & 4000 \\
\hline 2 & 1.1 & 4200 \\
\hline 3 & 0.6 & 4800 \\
\hline 4 & 2.0 & 5000 \\
\hline 5 & 0.6 & 5200 \\
\hline 6 & 1.9 & 5400 \\
\hline 7 & 1.4 & 6200 \\
\hline 8 & 0.9 & 6800 \\
\hline
\end{tabular}

Graph 2

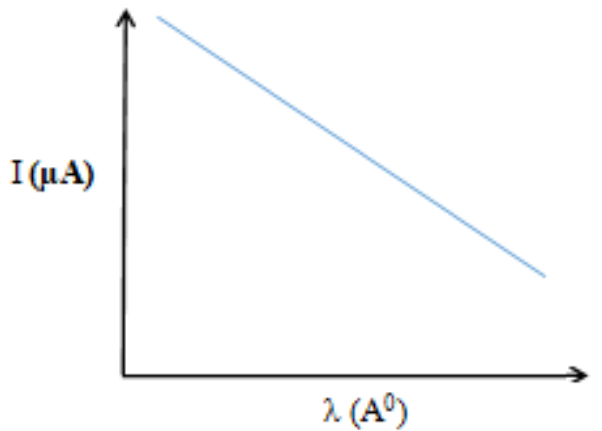


Table 2: To determine the absorption wavelength (different sized $(1,2,3)$ Carrot carbon quantum dots)

\begin{tabular}{|c|c|c|c|c|}
\hline S. No & $\begin{array}{c}\text { Wavelengh } \\
\lambda\left(\mathrm{A}^{0}\right)\end{array}$ & $\begin{array}{c}\text { Intensity } \\
1^{\text {st }} \text { size } \\
\text { particles } \\
(\mu \mathrm{A})\end{array}$ & $\begin{array}{c}\text { Intensity } \\
2^{\text {nd }} \text { size } \\
\text { particles } \\
(\mu \mathrm{A})\end{array}$ & $\begin{array}{c}\text { Intensity } \\
3^{\text {rd }} \text { size } \\
\text { particles } \\
(\mu \mathrm{A})\end{array}$ \\
\hline 1 & 4000 & 0.9 & 0.6 & 1.4 \\
\hline 2 & 4200 & 0.4 & 0.2 & 0.6 \\
\hline 3 & 4800 & 0.2 & 0.1 & 0.3 \\
\hline 4 & 5000 & 0.8 & 0.4 & 1.0 \\
\hline 5 & 5200 & 0.2 & 0.1 & 0.3 \\
\hline 6 & 5400 & 0.7 & 0.4 & 0.8 \\
\hline 7 & 6200 & 0.5 & 0.3 & 0.6 \\
\hline 8 & 6800 & 0.4 & 0.2 & 0.4 \\
\hline & & $\mathrm{I}_{\min }=0.2$ & $\mathrm{I}_{\min }=0.1$ & $\mathrm{I}_{\min }=0.3$ \\
\hline
\end{tabular}

Dips (i.e $I_{\min }$ for 1,2 and 3 sized particles are found at colors

\section{l. green $=520 \mathrm{~nm}$ \\ 2. violet $=480 \mathrm{~nm}$}

\section{B. Statistical analysis}

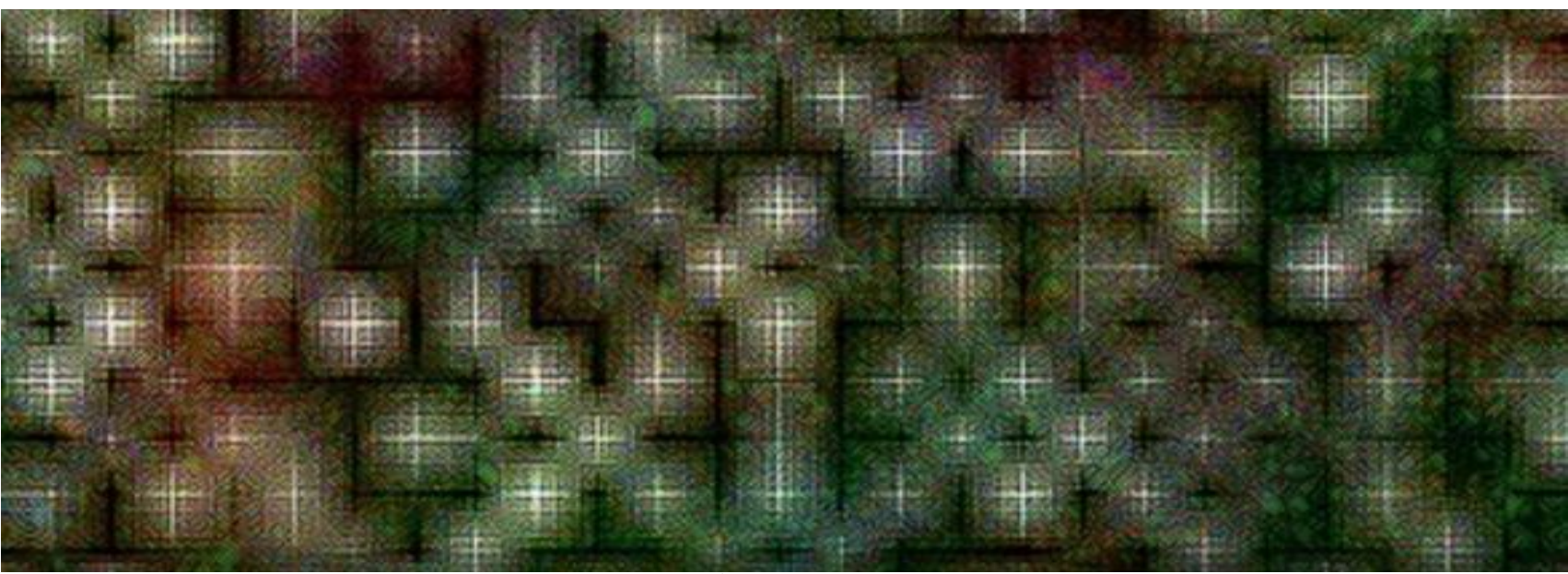

\section{$1^{\text {st }}$ magnification}

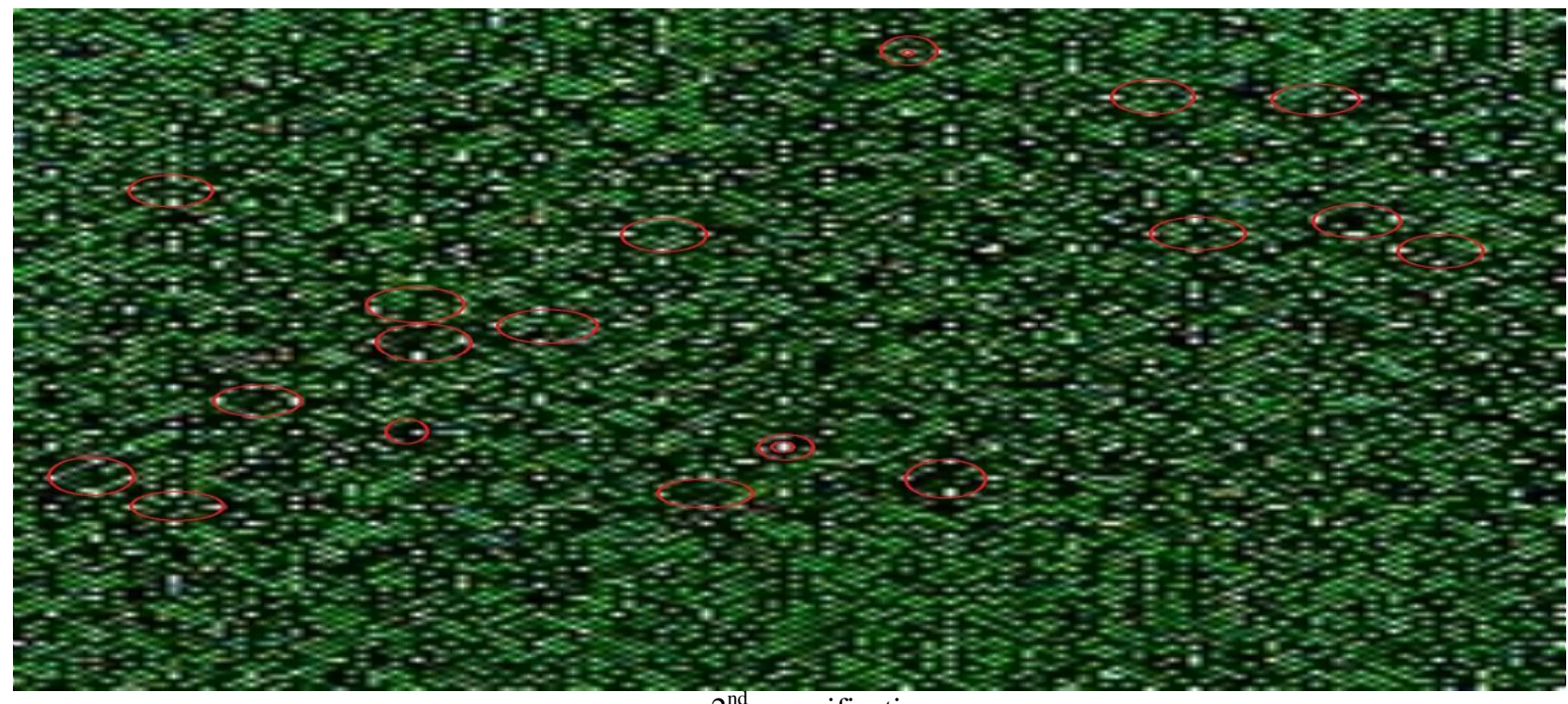

$2^{\text {nd }}$ magnification 


\section{Spectrum of Carbon}

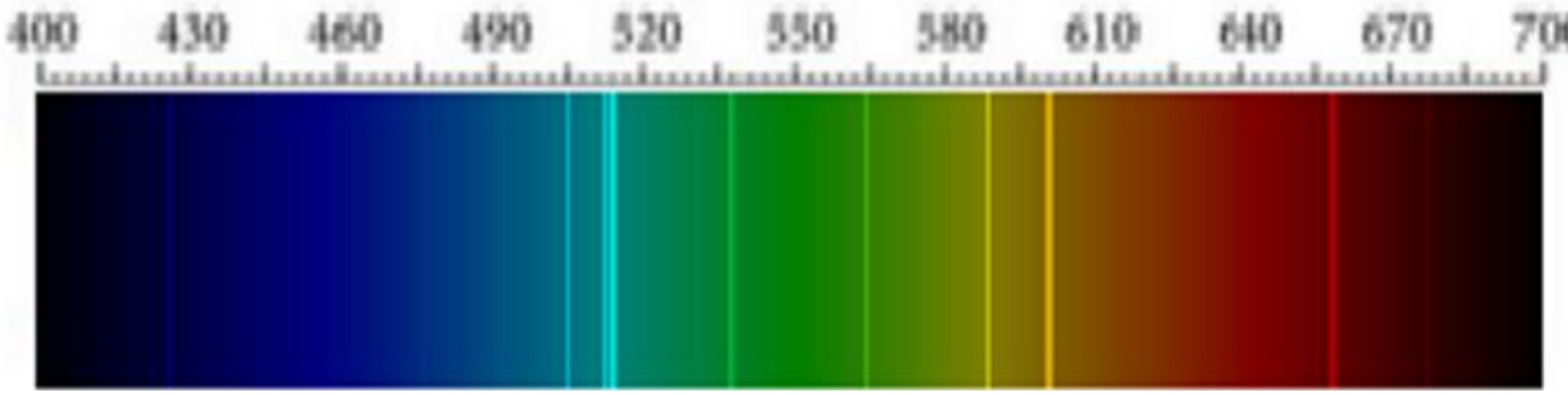

Table 3

\begin{tabular}{|c|c|c|c|c|}
\hline S. No & colours & $\begin{array}{c}\text { Number of counts } \\
(\mathrm{n}) \text { pxs }\end{array}$ & $\lambda(\mathrm{nm})$ & $\begin{array}{c}\% \\
\text { distribution }\end{array}$ \\
\hline 1 & Green2 & 21 & 562 & 18 \\
\hline 2 & Green1 & 59 & 538 & 51 \\
\hline 3 & Yellow & 10 & 589 & 9 \\
\hline 4 & Red & 9 & 658 & 8 \\
\hline 5 & Orange & 16 & 601 & 14 \\
\hline
\end{tabular}

\section{NO OF COUNTS}

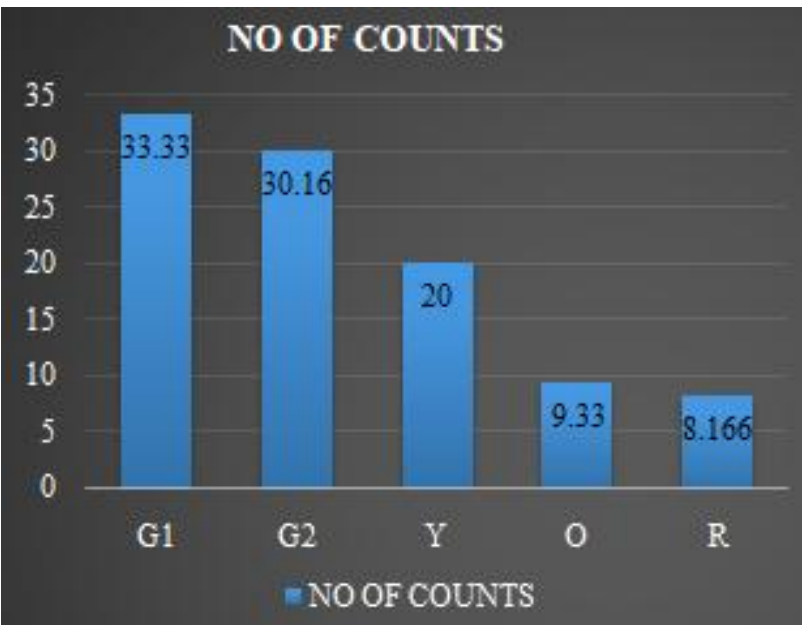

Graph 4
Result: intensity correlation

$$
\mathbf{I}_{\mathrm{G} 1}>\mathbf{I}_{\mathrm{G} 2}>\mathbf{I}_{\mathbf{0}}>\mathbf{I}_{\mathrm{Y}}>\mathbf{I}_{\mathrm{R}}
$$

\section{Laser Light Images}

\section{Laser diffraction}

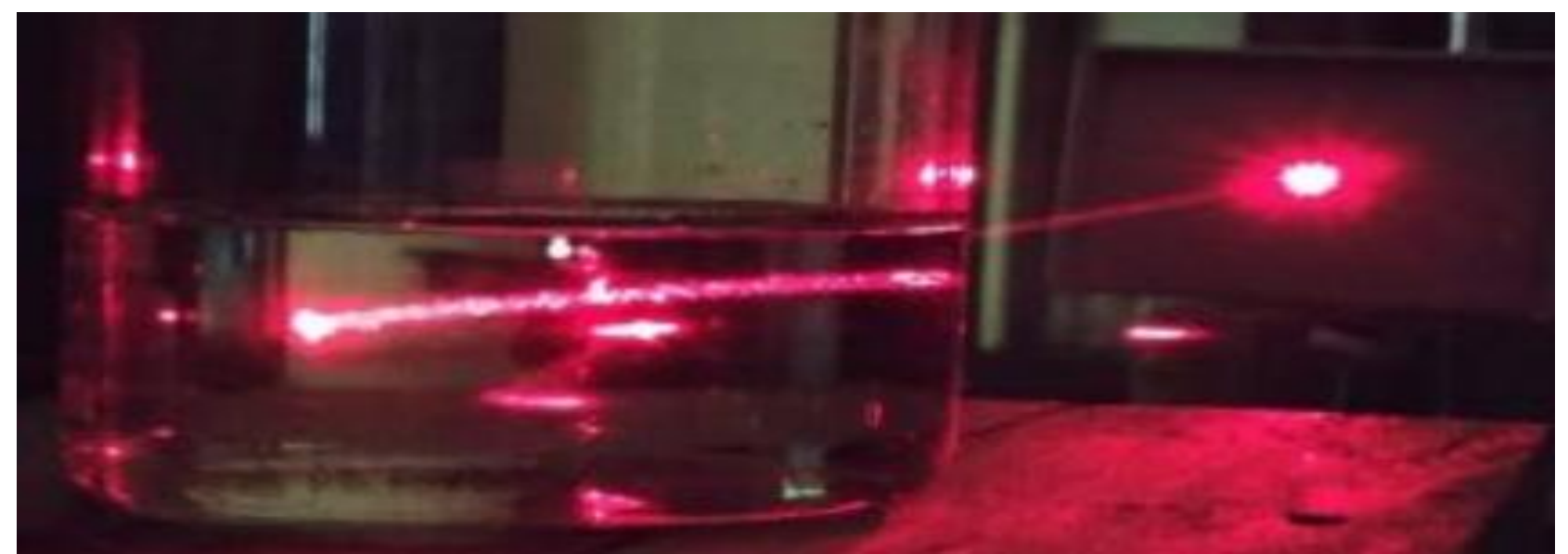

Laser diffraction pattern of HCQDs (lateral view) 

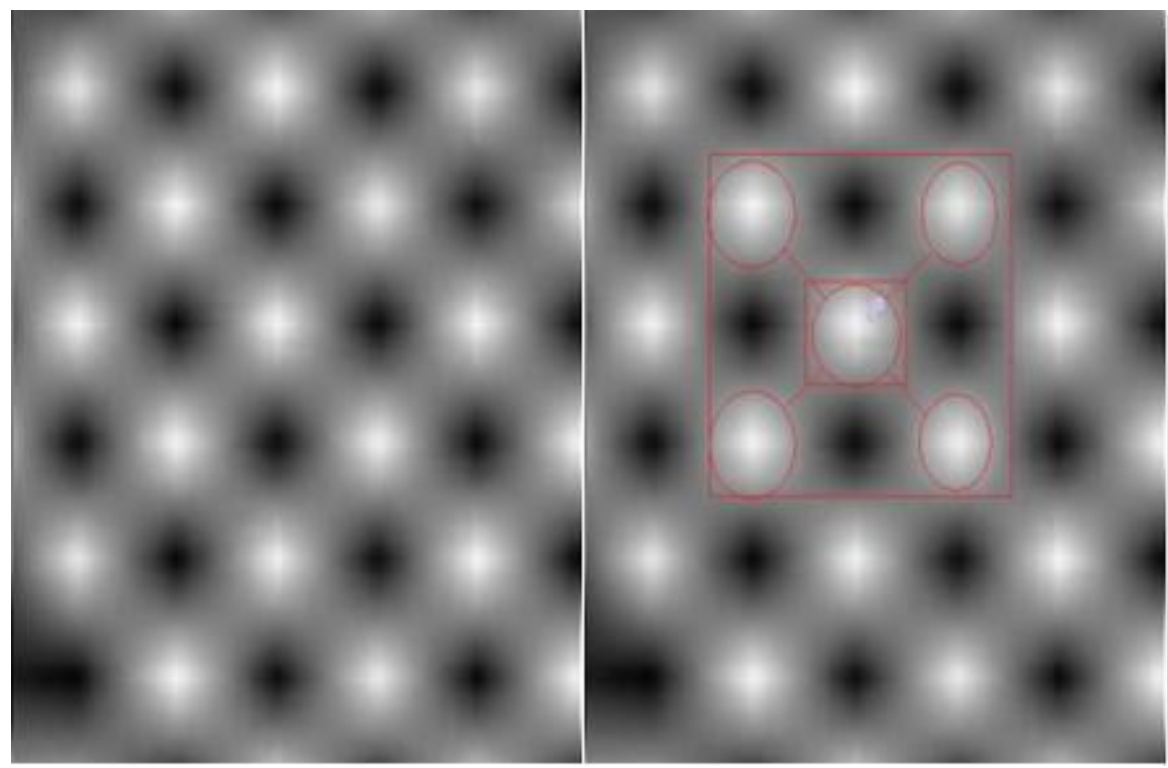

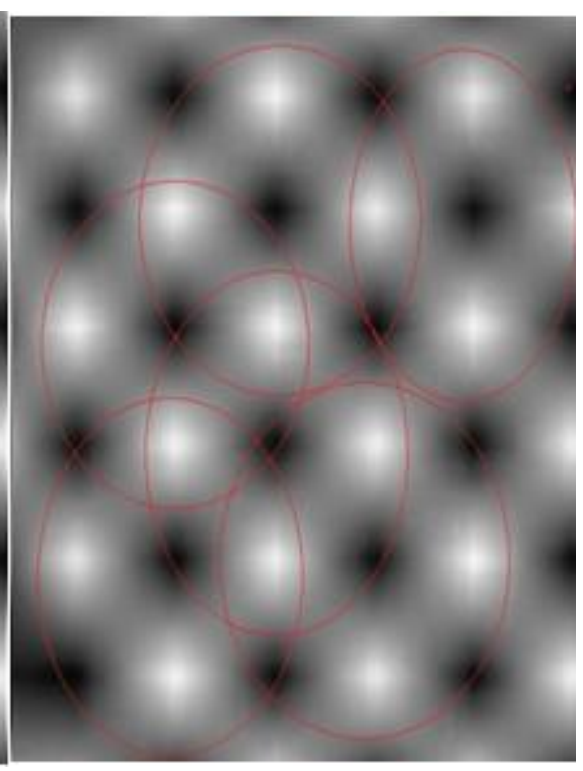

The higher order magnification of HCQDs shows the above structures, a regular pattern showing triclinic structure. Thus

1) CQDs and HCQDs are successfully prepared and their structural and optical properties are studied

2) CQDs show basic triclinic distribution

3) Maximum absorption occures at wavelength (green1) $480 \mathrm{~nm}$

4) These wavelengths matches with Raman carbon lines and frounhoffer lines. Thus experimental vales matches with theoretical vales .

5) Within available experimental facility we were able to identify the CQDs in micrometer ranges.

6) CQDs identified are of size $1400 \mu \mathrm{m}$ by first magnification method .Second magnification identifies CQDs of size $\mathbf{0 . 0 9 7 2 n m \& ~ t h e y ~ l o o k ~ g r e e n ~ i . e . ~}$ absorption/emission in green $\mathbf{5 6 2} \mathbf{n m}$

7) The statistical analysis by first magnification method gives the spectral intensity distribution correlation of CQDs as $\quad \mathbf{I}_{\mathbf{G 1}}>\mathbf{I}_{\mathbf{G} 2}>\mathbf{I}_{\mathbf{O}}>\mathbf{I}_{\mathbf{Y}}>\mathbf{I}_{\mathbf{R}}$,for green1(538nm), green2(562nm) ,orange $(601 \mathrm{~nm})$, yellow $(589 \mathrm{~nm})$, red $(658 \mathrm{~nm})$, with Percentage distribution as $51 \%, 18 \%, 14 \%, 9 \%, 8 \%$ for $\mathrm{G} 1, \mathrm{G} 2, \mathrm{O}, \mathrm{Y}, \mathrm{R}$ respectively

8) Laser diffraction pattern proves CQDs as coherent scattering centers and coherent carriers of electromagnetic energy in red wavelenth region with minimum loss in the surrounding medium.

\section{References}

[1] Carbon quantum dots from natural resource: A review Rashmita Das , Rajib Bandyopadhyay , Panchanan Pramanik https://doi.org/10.1016/j.mtchem.2018.03.003 /2468-5194/@ 2018 Elsevier Ltd. All rights reserved

[2] Green synthesis of fluorescent carbon dots from carrot juice for in vitro cellular imaging, Yang Liu1, Yanan Liu1, Mira Park2, Soo-Jin Park3, Yifan Zhang3, Md Rashedunnabi Akanda4, Byung-Yong Park4 and Hak Yong Kim/Carbon Letters Vol. 21, 61-67 (2017)/DOI: http://dx.doi.org/ 10.5714/CL.2017.21.061

[3] Carbon quantum dots from natural resource: A review /Rashmita Das, Rajib Bandyopadhyay, Panchanan Pramanik, Materials Today Chemistry $@ 2018$ Elsevier Ltd. All rights reserved.
[4] Aqueous Carbon Quantum Dot-Embedded PC60PC61BM Nanospheres for Ecological Fluorescent Printing: Contrasting Fluorescence Resonance EnergyTransfer Signals between Watermelon-like and Random Morphologies-Yu Jin Kim, Peijun GuoRichard D. Schaller/October 9, 2019/ https://doi.org/10.1021/acs.jpclett.9b02426 